\title{
Ueber eine neue Synthese des Guanidins ${ }^{1}$ ); von
}

G. Delitsoh.

Seit längerer Zeit bin ich mit der Untersuchung der Produkte beschäftigt, welche bei der Einktirkung von Chlorkohlensäureäther auf Sulfoharnstoff und Rhodanammonium entatehen. Ich erwartete, in ersterem Falle einen geschwefelten Allophansäureäther von der Zusammensetzung CS $\mathrm{NH}_{2}$

$\mathrm{NH}\left(\mathrm{COOC}_{2} \mathrm{H}_{5}\right)$, analog der Bildung von. Allophansäureäther aus Harnstoff, in letzterem Rhodenkohlensäureäther $\mathrm{CO} \underset{\mathrm{CNS}}{\mathrm{OC}_{2} \mathrm{H}_{5}}$ neben Salzsäure, bezüglich Chlorammonium zu erhalten: in beiden Fällen entsprach das Resultat den Erwartungen nicht, oder doch nur unvollständig. Bei der Einwirkung von Chlorkohlensäureäther auf Sulfoharnstoff bildet sich unter starker Erwärmung ein gut krystallisirtes Anlagerungsproduct aus gleichen Molektilen der augewendeten Körper, dessen Zersetzungsprodukte ziemlich complicirt sind. Aus. Rhodanammonium. seheint sich zwar Rhodankohlensäureäther zu bilden, worauf wenigstens die

1) Hr. Delitasch hat das Hauptergebniss seiner Versuche schon Bd. 8, S. 240 durch eine vorläufige Mittheilung zur Kenntniss gebracht. Als diese Mittheilung sich eben im Druck befand, erhielt ich von Professor Volhard aus München eine briefliche Mittheilung von seinen neueren, sebr interessanten Arbeiten üher Harnstoffe, aus der ich sah, dass Volhard und Delits ch bezüglich der Bildung von Guanidin ans Rhodanammonium zu gleicher Zeit dieselben Boobachlungen gemacht haben. Da jetzt von Beiden die ausführlicheren Mittheilungen ihrer Versuche mir zugegangen sind, so bringe ich dieselberl hinter einander zum Abdruck.

H. K.

Journal f. prakt. Chemie [2] Ba. $\theta$. 
2 Delitseh: Ueber eine neue Synthese des Guanidins.

reichliche Salmiakausscheidung und die Reaktionen des frisch gebildeten Aethers schliessen lassen, die Unbeständigkeit des letzteren aber machte mir bisher die Reinigung und Analyse unmöglich Die Zersetzungsprodukte desselben haben mit den aus oben erwähntem Anlagerungsprodukt erhaltenen grosse Aehnlichkeit, und hoffe ich, in kurzer Zeit über diese Körper Näheres mittheilen zu können.

Zu diesen Versuchen stellte ich grössere Mengen Sulfoharnstoff dar, den ich nach Reynold's Methode leicht erhielt; hierbei fiel mir indess ein Umstand auf, der mich fuir einige Zeit von meiner eigentlichen Arbeit ablenkte. Ich erhitzte das Rbodanammonium etwas zu hoch und erhielt aus der gesättigten Lösung beim Erkalten neben einer nur geringen Menge Sulfoharnstoff einen in dünnblättrigen, biegsamen grossen Krystallen anschiessenden Körper, welcher die Rhodanreaktion sehr scharf zeigte, mit Natronlauge in der Kälte aber kein Ammoniak entwickelte. Die Analyse ergab folgende Zahlen:

I. 0,491 Grm. Substanz mit chrnms. Blei verbrannt gaben 0,374 Kohlensäure und 0,229 Wasser.

II. 0,426 Grm. Substanz mit chroms. Blei verbrannt gaben 0,319 Kohlensäure und 0,199 Wasser.

III. 0,2705 Grm. Substanz mit chroms. Blei verbrannt gaben 0,207 Kohlensäure und 0,135 Wasser.

IV. 0,178 Grm. Substanz nach Carius mit rauchender Salpetersäure und chroms. Kali auf $230^{\circ}$ erhitzt, gaben $0,362 \mathrm{BaSO}_{4}$, entsprechend 0,0497 Schwefel.

In 100 Theilen

\begin{tabular}{lrrrrr}
\multicolumn{4}{c}{ berechnet } & \multicolumn{3}{c}{ gefunden } \\
$\mathrm{C}_{2}$ & 24 & 20,34 & 20,77 & 20,04 & 20,89 \\
$\mathrm{H}_{0}$ & 6 & 5,07 & 5,16 & 5,19 & 5,54 \\
$\mathrm{~S}$ & 32 & 27,13 & 27,92 & - & - \\
$\mathrm{N}_{4}$ & 56 & 47,46 & - & - & -
\end{tabular}

Nach der Analyse und den erwähnten Reactionen unterlag es kaum einem Zweifel, dass der schön krystallisirte Körper rhodanwasserstoffsaures Guanidin sei, zur 
Delitsch: Ueber eine neue Synthese des Guanidins. 3

Bestätigung wurde der Versuch wiederholt. In einem Kolben wurde Rhodanammonium im Oelbade auf etwa $220^{\circ}$ erhitzt, die entweichenden gasförmigen Producte durch eine gekühlte Vorlage mit wenig Wasser geleitet. Ströme von Ammoniak, welches nicht absorbirt wurde, entwichen, eine beträchtliche Menge Schwefelkohlenstoff sammelte sich am Boden der Vorlage. In Folge secundärer Bildung traten die charakteristischen Krystalle des von $\mathrm{Z}$ ois e beschriebenen trisulfocarbonsauren Ammoniaks auf, auch liess sich nach dem Verdunsten der wässrigen Lógung dieses Salzes in der Vorlage Rhodanammonium nachweisen.

Der Process, nach welchem die Bildung des Rhodanguanidins vor sich geht, scheint folgender ') zu sein :

$$
\begin{aligned}
& \operatorname{Cs}\left\{\begin{array}{l}
\mathrm{NH}_{2} \\
\mathrm{NH}_{2}
\end{array}\right. \\
& \operatorname{Cs}\left\{\begin{array}{l}
\mathrm{NH}_{2} \\
\mathrm{NH}_{3}
\end{array}=\mathrm{CH}\left\{\begin{array}{l}
\mathrm{NH} \\
\mathrm{NH}_{2} \mathrm{CSNH}+2 \mathrm{H}_{3} \mathrm{~N}+\mathrm{CS}_{2} \\
\mathrm{NH}_{2}
\end{array}\right.\right.
\end{aligned}
$$

1) So eben geht mir eine Abhandlung aus den Sitzungsberichten der physikaliseh-chemischen Classe der Akademie der Wissenschaften in München zu, worin Herr Professor Volhard gleichfalls über die Bildung von Rhodanguanidin aus Phodanammonium berichtet. Derselbe erklärt die Bildung des Rhodanguanidins durch folgende Gleichung :

$$
5 \mathrm{CNSNH}_{4}=2 \mathrm{CNSCN}_{3} \mathrm{H}_{6}+\mathrm{CS}_{3} \mathrm{~N}_{2} \mathrm{H}_{8}
$$

nimmt also die Bildung des Zeise'sehen Salzes als primär and hauptsächlich an. Ich kann mich dieser Ansicht nicht anschliessen. Die oben angeführte Bildungsgleichung des Rhodanguanidins lässt an Einfachheit niohts zu wünschen übrig, die entweichenden Produkte sind Schwefelkohlenstoff und Ammoniak, der Gewichtsverlust spricht gleichfalls für die obige Annahme, da der theoretische Gewichtsverlust 48,2\% betragen würde, und endlich hat das Auftreten von sulfocarbaminsau* rem and trisulforarbonsaurem Ammoniak durchaus nichts Befremdendes, da sich dasselbe bei der Berütirang von Schwefelkohlenstoff und Ammoniak bildet.

$$
\mathrm{CS}_{2}+2 \mathrm{NH}_{3}=\mathrm{CS}_{\mathrm{NH}_{2}}^{\mathrm{SNH}_{4}}+\mathrm{H}_{2} \mathrm{~S}=\mathrm{CS}_{\mathrm{SNH}_{4}}^{\mathrm{SNH}_{4}}
$$


4 Delitsch: Ueber aine neue Synthese des Guanidins.

Nach zweistüudigem Erhitzen des Rhodanammoniums auf $190^{\circ}--200^{\circ}$ beträgt der Gewiehtsverlust etwa $50 \%$; in der Schmelze fanden sich nur noch geringe Mengen von Sulfoharnstoff und Rhodanammonium.

Die Schmelze wird in Wasser gelost, filtrirt and durch Umkrystallieiren gereinigt. Zur Prüfung auf die Reinheit des Rhodanguanidin gewährt Thalliumoxydlösung ein vortreffliches Mittel. Die geringsten Spuren von Sulfoharnstoff, welche hartnäckig anhängen, geben damit sohwarze Flocken von Schwefelthallium, während reines Rhodanguanidin weisses Rhodanthallium fältt, das sich beim Erhitzen klar löst und nach dem Erkalten das Gefäss mit farblosen, metalliseh glänzenden Nadeln erfüllt.

Das Rlodanguanidin bildet grosse farblose, biegsame Krystallblätter, von eigenthümlichem Fettglanz; es ist leicht löslich in Wasser und Alkohol, schmilat bei $118^{\circ}$ and kann bis $150^{\circ}$ ohne Zersetzung erhitzzt werden. Bei weiterem Erhitzen tritt Zersetzung ein unter Ammoniakentwickelung, und Melam bleibt zuriick. Das geschmolzene Rhodanguanidin erstarrt in glänzeuden Krystallen. Durch nicht zu concentrirte Natronlauge wird das Rhodanguanidin nicht, oder doch erst nach langer Zeit angegriffen; Kalilauge entwickelt schon in der Kälte reichliche Mlengen von Ammeniak. Es entsteht zunächst Harnstoif, neben Khodankalium und Ammoniak:

$$
\left(\mathrm{CN}_{3} \mathrm{H}_{5}\right) \mathrm{CSNH}+\mathrm{KHO}=\mathrm{CON}_{2} \mathrm{H}_{4}+\mathrm{CSNK}+\mathrm{NH}_{3} \text {. }
$$

Durch weiteres Einwirken von Kalilauge zerfallt der Harnstoff in Ammoniak und Kohlensäure.

Durch Mineralsüuren kann die Rhodanwasserstoffsäure nicht direkt ersetzt werden, da sich Zersetzungsproducte bilden, welche sich auch durch wiederholtes Eindampfen, Ausziehen und Filtriren nicht entfernen lassen. Ich bebandelte darum das Rhodansalz in concentrirter Lösung mit salpetersaurem Kali, wobei salpetersaures Guanidiu und Khodankalium entsteht, dampfte zur Troekne ein und zog mit heissem Alkohol aus. In kaltem Alkohol ist das salpetersaure Guanidin sehr schwer löslich, so dass ich nach 
Delitsch: Ueber eine noue Synthese des Guanidins. 5

dem Abpressen und einmaligem Umkrystallisiren dasselbe völlig rein erhielt. Es wurde mit Salzsăure eingedampft und mit Platinehlorid in wässriger Lösung versetzt. Nach mässiger Concentration der Lösung sohossen die charakteristischen, orangegelben Nadeln des Platindoppelsalzes an, welohe abgepresst, getroaknet und der Analyse unterworfen wurden.

1,1085 Grm. Substanz gaben 0,416 Grm. Platin.

Die Formel $\left(\mathrm{CN}_{3} \mathrm{H}_{8} \mathrm{Cl}\right)_{2} \mathrm{PtCl}_{4}$ verlangt $37,32 \%$, gefunden $37,53 \%$.

Duroh Behandeln des rhodanwasserstoffauren Salzes mit Kupfervitriollösung erhält man leicht das schwefelsaure Guanidin, welches nach Ausfällen der Schwefelskure durch Barytwasser Guanidin liefert. Die Eigenschaften dieser Base sind schon von Hofmann genügend beschripben; es ist äusserst schwierig, das Guanidin völlig rein zu erhalten, da es mit grosser Begierde Kohlensäure aus der Luft anzieht. Man versetzt darum einfacher mit einem Ueberschuss von Barytwasser, leitet Kohlensäure bis zur vollständigen Sättigung ein und verdampft das Filtrat zur Trockne. Der Rückstand ist reines kohlensaures Guanidin, welches ein bequemes Material zur Darstellung aller andern Salze darbietet. Es krystallisirt in Formen des regulären Systems und ist äusserst leieht löslich in Wasser. Aus seiner wässrigen Lösung wird es durch Alkohol als Krystallpulver ansgeschieden.

Die Salze des Guanidins zeichnen sich dureh ihre ausserordentliche Krystallisationsfahigkeit, sowie durch ihre grosse Löslichkeit aus. Sie sind farblos, wenn die Säure farblos ist.

Das salzsaure Guanidin krystallisirt regulär und ist sehr zur Bildung salmiakartiger Verästelungen geneigt.

Das salpetersaure Guanidin ist von den Guanidinsalzen, die ich bis jetzt dargestellt habe, am schwersten löslich, macht aber immer, besonders in heissem Wasser, noch auf das Prädikat „leicht löslich“. Anspruch. Es krystallisirt in zarten weissen Krystallschuppen, welche oft zu langen 
6 Volhard: Ueber einige Derivate d. Sulfoharnstoffs.

blattähnlichen Gefügen aneinander gelagert sind und sich unter dem Mikroskop in zahllose Tetraëder mit abgestumpften Ecken auflösen

Das schwefelsaure Salz ist noch leichter löslich als die übrigen und erstarrt erst beim völligen Verdunsten seiner Lösung.

Besonders schön krystallisirt noch das chromsaure Guanidin, welches gedrungene, dunkel orangefarbene, glänzende Prismen bildet, welche treppenförmig übereinander gelagert sind.

Ich hoffe, in nächster Zeit ausführlioher ïher eine Reihe von Guanidinsalzen berichten zu können, besonders über das cyansaure Guanidin, welches zweifelsohne zun Guanidinharnstoff führt und mit dessen Darstellung ich jetzt beschäftigt bin. Die Darstellung des Rhodanguanidins aus Rhodanammonium ist für die nähere Kenntniss des Guanidins, dieser bisher noch so wenig untersuchten Base von nicht zu unterschätzender Wichtigkeit, da sie uns in den Stand setzt, das bisher ausserst kostspielige Material in jedem beliebigen Quantum ohne Mühe und bedeutende Kosten darzustellen.

Ueber einige Derivate des Sulfoharnstoffs ${ }^{\mathbf{1}}$; von

\section{J. Volhard.}

1. Senfölessigsäure.

Vor einigen Mouaten beschrieb ich in einer kurzen Notiz $\left.{ }^{2}\right)$ eine Verbindung, welche dureh Einwirkung von Monochloressigsäure auf Sulfoharnstoff entsteht, den Glycolylsulfoharnstoff oder das Sulfhydantoin. Ieh hatte die

1) Aus den Sitzungsberichten der mathem.-phys. Classe der k. Bayer-Akademie der Wissenschaften. 3. Januar 1874. 1.

3) Ann. Chew. Pharm. 166, 383. 